\title{
Uso de elementos de tierras raras contenidos en los discos duros de computadoras para optimizar la quema de combustibles fluidos ${ }^{1}$
}

\author{
Carlos Vallejos* y Job Balladares**
}

\section{Recibido: diciembre de 2012 / Aceptado: noviembre de 2014}

La investigación examinó el uso de imanes de neodimio provenientes de los discos duros de computadoras en desuso, y su utilización como optimizadores de la combustión de fluidos tales como propano, butano o la mezcla de los mismos. En la validación del efecto del campo magnético sobre los fluidos en referencia se utilizaron dos cocinas nuevas y dos cocinas previamente utilizadas, con su respectiva alimentación de gas. Los ensayos se realizaron sin y con imanes de neodimio provenientes de desechos tecnológicos. Como resultado de la investigación se concluyó que tales imanes son aptos para este propósito, con un rango de optimización en la combustión del gas de entre el 13 y el 20\%. Esto pudo demostrarse mediante la aplicación de principios generales de la mecánica de fluidos, del campo magnético y de la termodinámica. Los resultados contribuyen a la búsqueda de estrategias para disminuir el uso de combustibles fosiles en los hogares y otros espacios donde se utiliza gas licuado para la cocción de los alimentos, así cómo para contribuir al bienestar de la microeconomía.

Palabras clave: imanes /neodimio /discos duros /combustión /gas / campo magnético / desechos tecnológicos

1 Esta investigación participó en el Concurso de Emprendimientos Científicos y Tecnológicos convocado por la Organización de Estados Iberoamericanos para la Educación, la Ciencia y la Cultura (OEI) y el Ayuntamiento de Zaragoza en colaboración con la Fundación Zaragoza Ciudad del Conocimiento. Como resultado, fue una de las 19 propuestas seleccionadas por el jurado a partir de 76 postulaciones enviadas. El premio recibido consistió en una invitación a participar en el Congreso Iberoamericano de Ciencia, Innovación, Tecnología y Educación, celebrado en Buenos Aires, Argentina del 12 al 14 de noviembre de 2014.

* Facultad de Ciencia, Tecnología y Ambiente, Universidad Centroamericana. Rotonda Rubén Dario 150 mts. al oeste. Apartado 69, Managua, Nicaragua. Correo electrónico: carlosv@ns.uca. edu.ni

**Especialista en propiedad intelectual e inventor. Correo electrónico: jobballadares@hotmail.com 


\section{Introducción}

El neodimio es un elemento químico de la serie de los lantánidos de la tabla periódica, su símbolo químico es $\mathrm{Nd}$ y su peso atómico es 60 . Con este elemento de tierras raras se ha creado el imán más potente conocido hasta ahora. Su composición es $\mathrm{NdFeB}$, llamado imán de neodimio. El uso del neodimio como optimizador en la combustión de fluidos se remonta a los años 1885, cuando el sueco C. Auer von Welsbach inventó una novedosa camisa incandescente para gas. Como resultado de su invento, la llama de la cocina brillaba más y prolongaba la vida útil de los quemadores de gas. En 1936 los chinos comenzaron a utilizarlo para optimizar la quema de combustible en los motores de vehículos a base de gasolina.

En la actualidad el uso de este valioso metal de tierras raras tiene diversas aplicaciones. Se ha desarrollado una industria en fabricación de imanes, así como de dispositivos que optimicen los fluidos en su combustión (Rodrigues Flores, 2006). En el estado del arte también se cuenta con la publicación española número 1031990 (Ordóñez Maza, 1996) de la Oficina Española de Patentes y Marcas (OEPM). Ahí se divulgan los principios generales de la mecánica y efecto al que se somete un fluido cuando atraviesa un campo magnético.

Por lo tanto, la investigación retoma lo que se concentra en el estado del arte, con un enfoque tecnológico:

1- Los fundamentos hidráulicos nos dicen que cuando un fluido pasa por una tubería de diámetro mayor, y este diámetro se reduce, el fluido aumenta su velocidad, a la vez que se disminuye el volumen total suministrado, provocando un ahorro en volumen.

2- El fluido es sometido a un campo magnético, donde sufre un efecto ordenador y fraccionador de sus moléculas. En consecuencia, la quema de un fluido tratado así libera mayor calor de combustión que un fluido no tratado con un campo magnético de alta intensidad, como la que posee un imán de neodimio (Sagawa, Fujimura \& Matsuura, 1988; Croat, 1981).

Lo anterior es descrito en el documento de patente con número de publicación ES 1031990 U. Otro documento que se considera pertinente citar es la publicación ES 1071184 U (Espert Herrera, Forado Castiel, Sendra Marti \& Escobar Torres 2009), presentada ante la OEPM el 28/09/2009 y publicada el 03/02/2010. En sus antecedentes aparece más de una docena de documentos, desde solicitudes a través del Tratado de Cooperación en Materia de Patentes (PCT), hasta solicitudes USPTO de Inglaterra y Francia.

Sin embargo, cuando un gas pasa por un campo magnético se reduce la turbulencia del fluido, y aunque aumenta su poder de combustión por el ordenamiento de las moléculas y su fraccionamiento, disminuye su turbulencia, provocando en el quemador un flujo de calor con un movimiento menos estacionario que si el flujo fuese turbulento. Por esto se hace necesario diseñar un sistema que provoque cierta turbulencia cercana al quemador del fluido. El objetivo es que el calor de combustión del fluido fraccionado sea más estacionario en el quemador, 
provocando el aumento en la optimización de su calor de combustión. Un dispositivo podría lograr la aplicación de este nuevo aporte en la quema de combustibles fluidos tales como propano, butano, y mezclas de los mismos o similares (Serrano, 1996).

\section{Objetivos}

- A raíz de lo expuesto en el capítulo anterior, los objetivos del estudio fueron:

- Validar una tecnología de bajo costo que contribuya a la mitigación de problemas socio-económicos y ambientales.

- Evaluar la aplicabilidad de los imanes provenientes de discos duros de computadoras como optimizadores en la quema de gas butano y/o propano a través de experimentos realizados en el laboratorio.

\section{Materiales y método}

Los ensayos para comprobar la aplicabilidad de los imanes de neodimio se llevaron a cabo en el Laboratorio de Química de la Universidad Centroamericana (UCAManagua) institución patrocinadora de esta investigación, que se realizó en un período de tres meses.

Los ensayos que se llevaron a cabo son los siguientes:

\section{Ensayo 1:}

Se tomaron dos beakers de la misma marca y se llenó cada uno con $600 \mathrm{~mL}$ de agua. Estos dos beakers se colocaron en el quemador de dos cocinas previamente utilizadas. Uno se calentó con el dispositivo del imán de neodimio (instalado a 2 cms. de distancia de la conexión a la válvula de la cocina) y el otro sin neodimio. Se calentó el tiempo necesario hasta evaporar los $600 \mathrm{~mL}$ en cada uno de los beakers.

\section{Ensayo 2:}

Se instalaron dos cocinas nuevas, con válvulas nuevas, marca Eco, de dos quemadores. Se procedió a medir $300 \mathrm{~mL}$ de agua. El agua se calentó hasta su ebullición y evaporación durante 10 minutos, luego se dejó enfriar y se midió en una probeta el volumen de agua que quedó de la evaporación. Por diferencia de volumen inicial de agua menos el volumen final se obtuvo el volumen de agua evaporado. El volumen evaporado fue de $124 \mathrm{~mL}$.

Se procedió a realizar el experimento anterior, pero la segunda vez se instaló un par de imanes de neodimio de forma irregular - tal como se muestra en la Ilustración 1 - sobre el ducto del gas, en la parte superior a $2 \mathrm{~cm}$ de la salida del fluido. Se midió la diferencia de volumen para compararlo al obtenido sin neodimio instalado. No se encontró diferencia. El resultado fue de $124 \mathrm{~mL}$ de agua evaporados.

Se tomaron $600 \mathrm{~mL}$ de agua y se pusieron a calentar hasta su ebullición durante 20 minutos, luego se enfriarion por 10 minutos. El ensayo se repitió tres veces con una media de volumen evaporado de $234 \mathrm{~mL}$ de agua. Los ensayos para tomar la media fueron realizados cinco veces. Este calentamiento se realizo en un recipiente metálico y en una cocina nueva y sin imanes de neodimio. Posteriormente 
se procedió a hacer el mismo ensayo en una cocina nueva y con imanes de neodimio, el rendimiento de la cocina dio una evaporación promedio de $318 \mathrm{~mL}$ de agua. El ensayo se repitió cinco veces.

\section{Ensayo 3:}

En las mismas condiciones que los ensayos anteriores, en una cocina a la que se le instalaron dos imanes de neodimio, con la llama completamente baja, y con un tiempo de 20 minutos. El agua evaporada fue mayor en un 10\% en volumen en la cocina a la que se aplicó el $\mathrm{Nd}$. Estos resultados demuestran que el mayor ahorro de combustible se da cuando la llama está baja.

\section{Ensayo 4:}

Un volumen de $300 \mathrm{~mL}$ de agua fue llevado hasta su ebullición durante 10 minutos en una cocina nueva en la que nunca se había utilizado neodimio. Se enfrió el líquido durante 10 minutos y se midió en una probeta, luego se obtuvo la diferencia entre el volumen inicial y el volumen final.

El mismo ensayo se realizó en una cocina diferente en la que se instaló un par de imanes de neodimio sobre la manguera que suministró el gas.

Luego se experimentó el calentamiento y evaporación en un tiempo de 20 minutos, 30, 40 y 50 minutos, para lo que fue necesario trabajar con 600 y 1000 $\mathrm{mL}$ de agua.

El principio científico de la experimentación es que el calor suministrado por la llama es directamente y aproximadamente igual a la cantidad de mililitros de agua evaporada, y que si el Neodimio aumenta el poder calorífico del gas, mediante el ordenamiento y fraccionamiento de las moléculas del gas, en iguales condiciones termodinámicas, para que exista un ahorro en combustible al reducir el diámetro por el cual pasa el gas, el volumen de agua evaporada debe por lo menos ser igual en ambos ensayos. $\mathrm{Q}=\mathrm{mCp} \Delta \mathrm{T}$; donde $\mathrm{Q}$ ganado es igual $-\mathrm{Q}$ cedido.

\section{Resultados y discusión}

Los resultados, acompañados de su discusión, se presentan a continuación para cada ensayo.

\section{Ensayo 1:}

Ambos beakers se calentaron hasta evaporar los $600 \mathrm{~mL}$ de agua. Durante el experimento se observó que el beaker calentado en la cocina con dispositivo de neodimio sufrió una leve fisura (Ilustración 1). También se notó que los 600 $\mathrm{mL}$ se evaporaron más rápidamente en el beaker calentado en la cocina con los imanes de neodimio (Ilustraciones 2 y 3). Este experimento se realizó dos veces y los resultados fueron similares. Esto solamente se explica con el efecto del campo magnético del neodimio en el ordenamiento de las moléculas de hidrocarburos para una mejor combustión. Esta intensidad del campo magnético provocado por imanes de neodimio nos indicó que el experimento no se podía realizar en recipientes de vidrio, sino que en recipientes de un material más resistente al calor, por ejemplo, de acero inoxidable. 


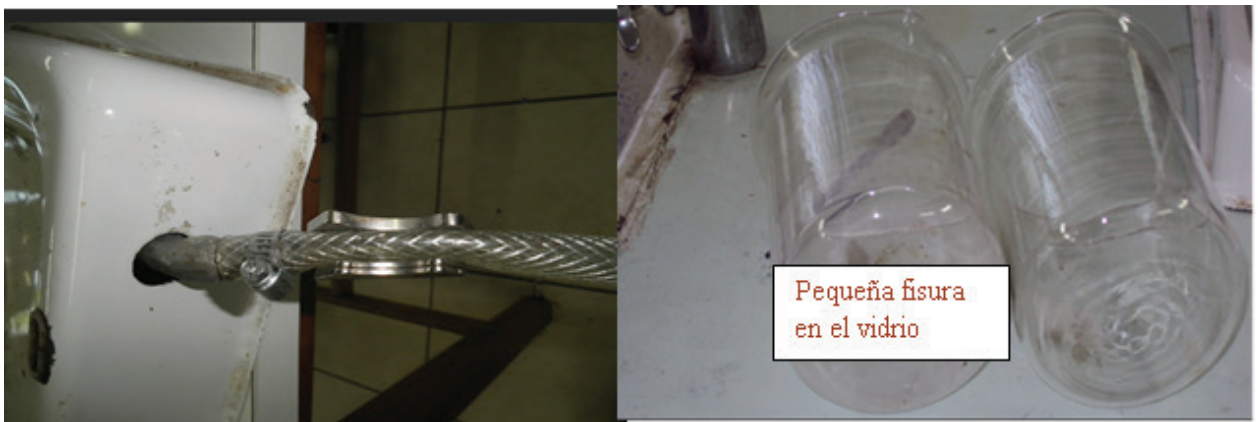

Ilustración 1. Instalación del iman de neodimio sobre la manguera que atraviesa el fluido y fisura en el beaker

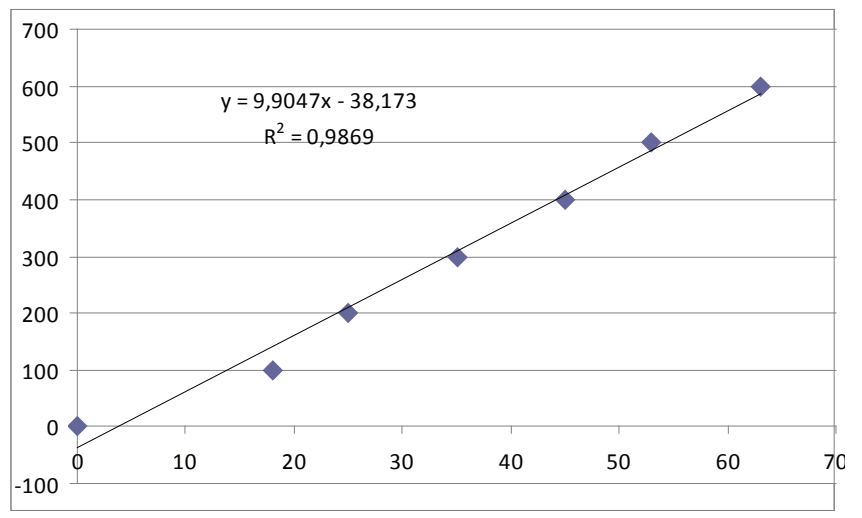

$\begin{array}{lc}\begin{array}{l}\text { Tiempo } \\ (\text { min) }\end{array} & \begin{array}{c}\text { mL de agua } \\ \text { evaporada }\end{array} \\ 0 & 0 \\ 18 & 100 \\ 25 & 200 \\ 35 & 300 \\ 45 & 400 \\ 53 & 500 \\ 63 & 600\end{array}$

Ilustración 2. Volumen de agua evaporada vs. tiempo utilizando imanes de neodimio

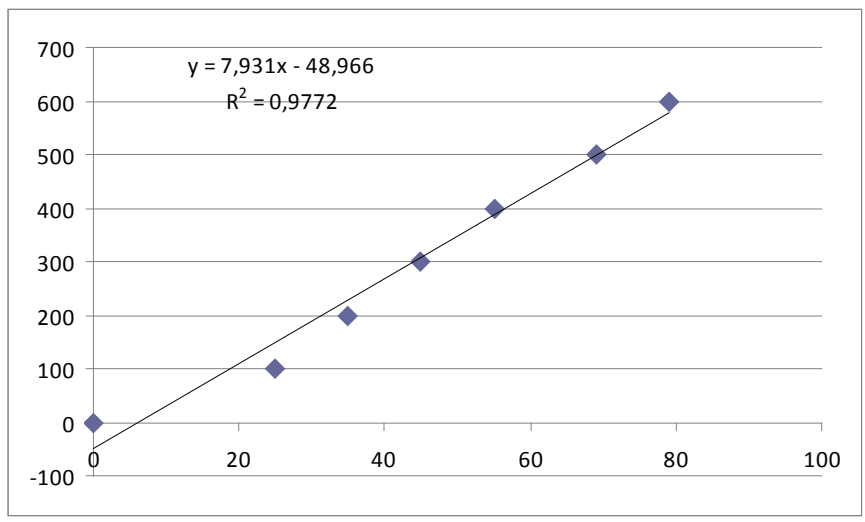

$\begin{array}{ll}\begin{array}{l}\text { Tiempo } \\ \text { (min) }\end{array} & \begin{array}{l}\text { mL de agua } \\ \text { evaporada }\end{array} \\ 0 & 0 \\ 25 & 100 \\ 35 & 200 \\ 45 & 300 \\ 55 & 400 \\ 69 & 500 \\ 79 & 600\end{array}$

Ilustración 3. Volumen de agua evaporada vs. tiempo sin imanes de neodimio

\section{Ensayo 2:}

Lo observado en este ensayo se debe a que el pase del gas puede estar estrangulado por la fuerza de atracción entre los imanes. El ahorro se refleja en volumen, el gas suministrado aumenta su poder calorífico para poder compensar el 
porcentaje de volumen ausente en el quemador. Si este dispositivo se instalara en un motor de auto para optimizar la quema de combustible y se estrangulara el pase del gas aún más, sería afectada negativamente la potencia del auto.

Al medir el volumen de gas suministrado en cada uno de los experimentos, se encontró que el gas que había sido suministrado con $\mathrm{Nd}$, y estrangulado su pase, tenía un volumen de aproximadamente un $18 \%$ menos que el gas suministrado sin $\mathrm{Nd}$, no estrangulado, lo que señala un ahorro en volumen de gas.

El ensayo se repitió cinco veces, dando resultados promedios a los del primer ensayo.

\section{Ensayo 3:}

La optimización del gas se mide por la diferencia de las cantidades de agua que se evapora. Donde hay más agua evaporada en igual tiempo y condiciones, hay más calor de combustión del gas, pero no necesariamente tiene que ser mayor la cantidad de agua evaporada. Basta que sea igual en igual tiempo y condiciones termodinámicas pues el paso del fluido está siendo estrangulado y, por lo tanto, a igual tiempo e igual cantidad de volumen de agua evaporado, existe una reducción en el suministro de gas en la manguera con neodimio. Mediante el principio de equilibrio térmico se explica que el calor suministrado es igual a la masa por el calor específico, en este caso el agua, por la diferencial de temperatura. La siguiente ilustración muestra las placas de neodimio utilizadas, así como un diseño del dispositivo magnético.
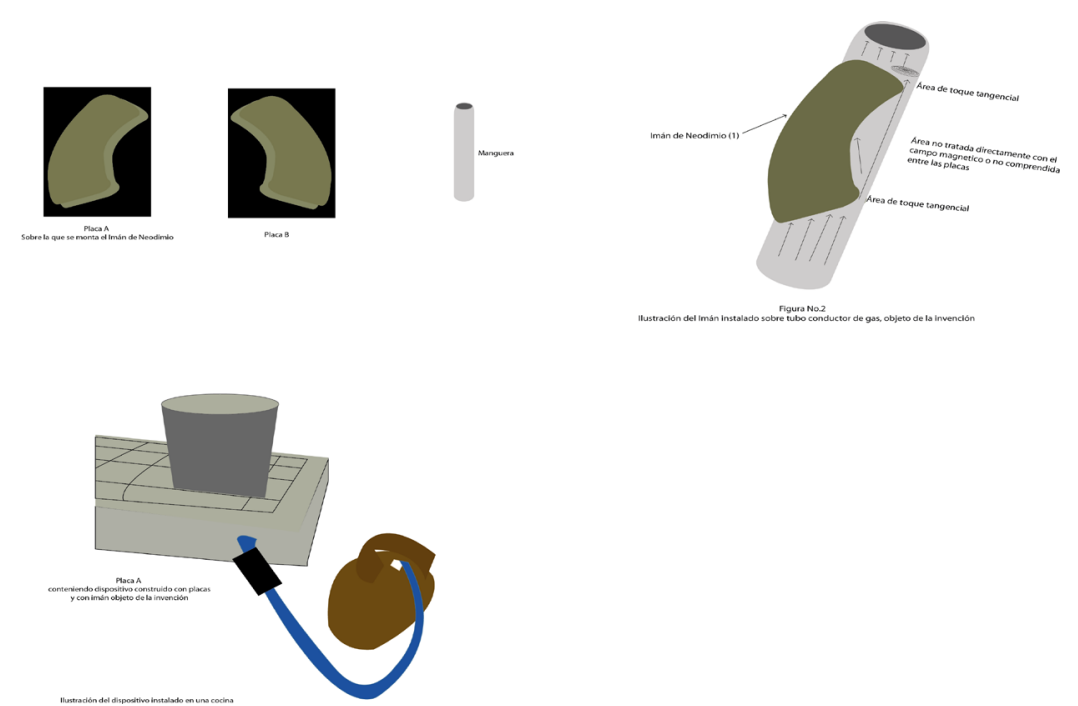

Ilustración 4. Placas A y B del dispositivo del imán de neodimio conectado a la cocina

\section{Ensayo 4:}

En varios de los ensayos la optimización en la quema del combustible gaseoso no fue apreciable. Por ejemplo, se observó que a iguales condiciones de ensayo se obtenía igual resultado con o sin Neodimio, lo que lleva a pensar que no todos los imanes de neodimio provenientes de las computadoras son capaces de ejercer un efecto magnetizante sobre las moléculas del gas. El volumen de agua evaporada era 
igual o ligeramente menor al utilizar Neodimio que al no utilizarlo. Por ejemplo, una diferencia de 2 o 10 y hasta $50 \mathrm{~mL}$ en medias de 700 o $900 \mathrm{~mL}$, en ensayos $1000 \mathrm{~mL}$ de agua al ser evaporados a 40 y 50 minutos, según el ensayo. En algunos ensayos el resultado era positivo, obteniéndose una reducción en el tiempo de ebullición e incremento en el volumen de agua evaporada hasta de un $25 \%$ más con imanes de Neodimio que sin imanes de Neodimio, lo que hacía obvio el efecto del campo magnético sobre el gas. Sin embargo, imanes que daban resultados en una cocina aparentemente no lo daban en otra. Por ello, se procedió a investigar si existía un problema en la confección de las válvulas o los quemadores. No obstante, no se encontró fallas en las válvulas ni se pudo constatar fallas en los quemadores. Por lo tanto se procedió a realizar el ensayo con la llama más baja que la cocina podía dar, obteniéndose un dato positivo de aproximadamente el $10 \%$, lo cual significaba que existía un ahorro sobre la llama más baja para la cual se diseñó la cocina. Pero aún no se podía entender por qué.

Se procedió, por lo tanto, a hacer uso de pesas a fin de observar una disminución del peso al utilizar Neodimio y comparar esa disminución con el ensayo en el que no se utilizó Neodimio. No se pudo detectar diferencia debido a que no se contaba con una pesa calibrada donde se pudiera leer decimales. Tampoco se contaba con un medidor de fluidos, por lo que se procedió a medir la cantidad de fluido suministrado con y sin Neodimio a través del uso de un cuerpo inflable, como el de un globo. Sin embargo se contactó que la válvula utilizada para dispensar el fluido con Neodimio no poseía la presión suficiente para inflar el globo, por lo que se procedió a quitar el Neodimio y luego se suministró el gas, constatándose que tampoco posee la capacidad para vencer la resistencia de llenado del globo.

Por último se procedió a hacer un experimento comparativo a través de la utilización de otro fluido que pudiese ilustrar lo que estaba pasando para poder apreciar la especulación razonable: "el Neodimio debe ejercer un efecto positivo sobre el gas".

Se instaló a una fuente de flujo de agua una manguera de las utilizadas en los ensayos, de un centímetro de diámetro y aún teniendo el par de imanes de Neodimio instalados. Se procedió a realizar mediciones de volúmenes de agua a intervalos de tres minutos, constatándose un flujo aproximadamente constante, con una velocidad desconocida y una reducción del diámetro también desconocida. Para solucionar este problema se consideró un área de pase del fluido reducida en un $50 \%$, bajo la consideración de que a mayor reducción en el diámetro se podría provocar un aumento del tiempo necesario para realizar la cocción normal de los alimentos al que el consumidor está acostumbrado.

Se encontró que el volumen obtenido en un periodo de nueve minutos fue de $30260 \mathrm{~mL}$, tiempo equivalente a 540 segundos. Al aplicar la ecuación general de los fluidos para el cálculo del flujo de volumen, resulta de aproximadamente $142 \mathrm{~mL} / \mathrm{s}$.

Pero ¿cuánto varía el volumen en relación a una manguera que no está siendo estrangulada con Neodimio? Era éste el punto crucial de la investigación.

Se procedió a quitar los imanes instalados en la manguera y se midió el flujo en igual tiempo, constatándose en muestras diferentes un flujo de $36280 \mathrm{~mL}$ en 540 segundos. Al hacer una relación porcentual se obtiene que el flujo sin Neodimio es mayor en igual tiempo pues el área de suministro del fluido es mayor, con un 
porcentaje aproximado al $18 \%$ más que cuando el Neodimio está estrangulando el agua. Esto es acorde al cambio del diámetro de pase del fluido de $855426 \mathrm{~mL} / \mathrm{s}$.

Dado que algunos ensayos con Neodimio y otros sin Neodimio proporcionaron los mismos resultados, realizándose en iguales condiciones, la diferencia de que al usarse Neodimio se estranguló el diámetro de suministro de gas debido al principio magnético, muestra que el Neodimio aumenta el efecto calórico en la combustión del gas en una relación porcentual equivalente al ahorro de combustible, o sea de $18 \%$. Con esto se hace obvio que hay un ahorro en volumen a la vez que hay un aumento del poder calórico del gas para que se pueda evaporar igual cantidad de agua con menos gas en igual cantidad de tiempo.

\section{Conclusiones}

En base a los resultados del trabajo de investigación realizado se concluye que:

1. Se consta con un dispositivo magnético para optimizar la combustión de fluidos tales como gas propano, butano o mezclas de los mismos. Éste está constituido por una placa A y una placa B a las que se adhieren los cuerpos magnéticos de manera tal que se atraen por igual en todos sus puntos. Dichas placas pueden ser metálicas o no metálicas, revestidas por un material aislante del campo magnético y con medios de sujeción suficientes para su instalación en un ducto cilíndrico transportador de fluidos. Sus imanes son en forma de C truncada y al menos una de sus partes extremas posee mayor anchura que su parte media. Están unidos en el punto medio de su parte externa izquierda en un ángulo de entre 25 y 45 grados, el cual estrangula de manera transversal el diámetro del pase del fluido desde un punto máximo cercano al vértice a un punto menor ubicado en el centro del ducto.

2. Conforme estrangula el pase del fluido, este dispositivo magnético forma un ángulo de entre 25 y 45 grados desde un punto máximo -parte superior cercana a la entrada del fluido al equipo de combustión- hasta un punto menor, ubicado en una sección longitudinal inferior del ducto. Al comprimir el gas en un ángulo de 25 a 45 grados, de manera horizontal o longitudinal, pueden obtenerse los rendimientos óptimos siempre que no se exceda de un 50\% de reducción del diámetro de pase del fluido.

3. El uso de un imán de tierras raras, Hierro y Boro en forma de C truncada o media luna truncada, y con al menos una de sus partes extremas con mayor anchura que su parte media es la novedad que se reivindica en esta investigación.

4. El imán proveniente de los discos duros de computadoras es apto para la instalación en cocinas de dos y tres quemadores, con las cuales se realizó el ensayo. El efecto pudo apreciarse de dos maneras, lo que implica que puede ser doble. Por ejemplo, el incremento en el 5\% del agua evaporada más el ahorro en el volumen del gas puede redondear el 23\% pues aumentar el agua evaporada no implica que no se esté ahorrando combustible. Esto lo explica la aplicación de la teoría general de la mecánica de fluidos, la cual se ensayó y demostró de manera práctica en el laboratorio. 
5. Dada la fuerza de presión instalada en la válvula, que no es capaz ni de inflar un globo, no se incurre en peligros de explosión por cerrar un 50\% o menos su pase. Una explosión podría ser producto de fuga en el tanque, sobrecarga del tanque, o la ignición de una chispa al tiempo que exista una fuga considerada, pero no producto de la compresión por Neodimio, ni por reducción del diámetro ni por ambos. La razón es que la manguera es lo suficientemente fuerte para soportar esta presión. Sin embargo, si el material de la manguera es muy maleable debe evitarse hacer presión en exceso pues se puede obstruir por completo el paso del gas.

6. Se demostró con esta investigación que efectivamente usando imanes de Neodimio se puede mejorar la efectividad de la combustión en la cocina. Estos imanes, que están contenidos en los discos duros de las computadoras, son desechos tecnológicos que podrían reutilizarse y ser potenciados como ahorrantes de combustibles.

7. Se comprobó la información disponible en bases de datos de patentes, donde se afirma que el imán de Neodimio-Boro-Hierro produce un campo magnético al interactuar con las moléculas de gases de los hidrocarburos.

\section{Agradecimientos}

Esta investigación fue posible gracias al apoyo de la Dirección de Investigación y Proyección Social de la Universidad Centroamericana a través de los fondos concursables que administra, en su edición de 2011. También mis sinceros agradecimientos al Ing. Job Balladares, quien ha motivado mi interés por la innovación y fue el promotor de esta idea innovadora, que juntos logramos concretar.

\section{Referencias bibliográficas}

Croat, J. J. (Sterling Heights, MI). (1981). High coercivity rare earth-iron magnets.

US4496395 A. (CA1202864A1). 29 Jan 1985. appl. 06/274,070, 16 Jun. 1981, 65p.

Espert Herrera, L., Forado Castiel, J., Sendra Marti, J. M. \& Escobar Torres, M. (2009). Aparato orientador de las moléculas de un fluido circulante. ES 1071 184, F02M 27/04 (2006.01). 3 Feb. 2010. U 200901368, appl.20 Sep. 2009, 8p.

Ordóñez Maza, F. (1995). Inductor ecolo-magnético. ES 1031 990. (Cl F02M 27/04) Solicitud de patente No. U 9501387, 16 March 96. U 9501387, appl.19 March 1995, 6 p.

Rodríguez Flores, R. (2005). Dispositivo magnético de neodimio en 10000 Gauss para mejorar la combustión del gas natural y gas propano o butano. MX NL05000004 A. (Cl. F17D 01/065), 5 Jul 2006, NL05000004, appl. 6 Jan 2005, 8 p.

Sagawa, M. (Nagaokakyo, JP), Fujimura, S. (Kyoto, JP) \& Matsuura, Y. (Ibaraki, JP). (1987). Magnetic materials and permanent magnets. US 4,770,723, (Cl. 148/302 ). 13 Sep 1988. 07/013,165, appl. 10 Feb.1987, 32 p.

Serrano, P. (1996). M.P. Unidad magnética para fluidos. ES 1033 463U. (Cl.: F23K 5/18.) 01 Sep 96, appl. U 9600684. 14 March 96, 6 p. 